\title{
Long-Term Reliability of Brittle Materials: The Issue of Crack Arrest
}

\author{
Yann Charles, ${ }^{1}$ François Hild ${ }^{1 *}$ and Stéphane Roux ${ }^{2}$ \\ ${ }^{1}$ LMT-Cachan, ENS de Cachan/CNRS-UMR 8535/Université Paris 6 \\ 61 avenue du Président Wilson, F-94235 Cachan Cedex, France. \\ ${ }^{2}$ Laboratoire "Surface du Verre et Interfaces", UMR CNRS/Saint-Gobain, \\ 39 quai L. Lefranc, F-93303 Aubervilliers Cedex, France.
}

\begin{abstract}
In structures containing brittle materials, residual and/or heterogenous stresses may prevent cracks to propagate up to failure. Consequently, for such structures, crack arrest has to be accounted for and a weakest link hypothesis may not be applicable. A probabilistic crack propagation model is derived to describe instantaneous or delayed arrest phenomena. A timedependent regime is induced by slow crack growth experienced by ceramics and glasses. A general expression is obtained in which instantaneous up to infinite propagation times can be modelled in a unified way. The results are illustrated on a case study dealing with propagation of cracks in a thin walled tube submitted to a temperature gradient through its thickness. Different types of propagation/arrest regimes can be identified.
\end{abstract}

Keywords: Crack propagation and arrest, Ceramics and glasses, Fracture toughness, Microstructures, Probability and statistics.

*corresponding author, Fax: +33 1474022 40, Email: Francois.Hild@lmt.ens-cachan.fr 


\section{INTRODUCTION}

The failure of brittle structures is generally caused by randomly distributed defects induced by the manufacturing process. To predict their ability to survive under a given stress field, a probabilistic approach is used. In many cases, the weakest link hypothesis is made [1] and the Weibull model [2,3] is applied: if one of these initial defects starts to propagate, then the whole structure breaks. This approach, describing the inception of crack propagation caused by initial defects [4], has been progressively extended to account for various statistical distributions of bulk or surface defects [5], multiaxial criteria for critical loads on defects [6,7], inhomogeneous stress fields [8]. The very heart of the formulation is restricted to the onset of propagation, and thus the basic hypothesis is that as soon as a defect becomes locally unstable, its propagation and the subsequent failure of the structure is postulated to occur with certainty.

However, there are numerous cases where a brittle material - because of its heterogeneity — or a structure - because of its shape and the type of loading - may sustain the presence of a crack. In such cases, the standard "Weibull approach" appears to be inadequate. For instance, fiber-reinforced composites have been introduced to avoid premature failure of a brittle matrix when it is reinforced by strong fibers [9]: extensive matrix-cracking can be observed prior to the final failure induced by fiber breakage. The onset of matrix-cracking can be described by a Weibull approach: the first fiber fracture event can be determined with the weakest link assumption. Another example concerns the fragmentation of impacted ceramics where many cracks are created prior to the failure of the material $[10,11]$.

This work is an attempt to address crack arrest, by taking into account the heterogeneity of the microstructure as well as a potentially inhomogeneous stress field, so that one can quantify the probability that a crack may propagate up to a prescribed length and stop. Moreover, aiming at the description of crack propagation in ceramics or glass $[12,13]$, subcritical growth of cracks will be considered $[14,15]$, so that the probability for a crack to reach a given length will be studied as a function of time. Applications concern for instance cracks 
initiated from indentations, where the residual stress field drives the crack extension, or complex loadings where a compressive stress field will lead to crack arrest in metal/ceramic assemblies [16]. It can be noted, that within the weakest link framework, models have been developed to evaluate the time-dependent reliability of brittle materials $[17,18]$. The latter is generally referred to as fatigue $[19,20]$, be it static (i.e., when the failure probability evolves with time under a constant applied load), dynamic (i.e., when the failure probability is stress rate sensitive) or cyclic (i.e., as usually known for metals and alloys).

\section{DEFINITION OF THE MODEL}

To address probabilistic crack arrest, one needs to consider 3D crack propagation in a heterogeneous material. 3D calculations are complex and detailed analyses tend to become rapidly untractable. One may mention the numerical work of Bower and Ortiz [21,22] on the propagation of a crack across a medium with tough particles. This approach is extremely valuable to get some insight into the full complexity of the by-passing of obstacles by a crack front, however, the numerics involved is too demanding to get an extensive statistical analysis of the crack propagation. Curtin $[23,24]$ performed a simplified analysis of a similar problem. A modeling of planar crack propagation in a random environment was also proposed by Schmittbuhl et al. [25], and Ramanathan et al. [26] which revealed a complex spatio-temporal organization of the propagation, and scale-invariant crack front morphology. These studies show that the macroscopic toughness is not simply given by average toughness and that tougher elements impede crack growth in a lesser extend than with a strongest link assumption due to the curvature of the crack at those sites which increases locally the stress intensity factor. In the following, we will develop a simplified model where the crack front is parametrized by a single parameter, i.e., its mean position. This simplication hypothesis will allow for a full analytical treatment of the crack propagation statistics. However, we need to discuss first the conditions under which such a simplified picture may be connected to the complete 3D problem. We are left here with two options: the first is a pure two- 
dimensional situation, where the considered medium is a thin plate with one single grain accross the thickness. In this case obviously, only the mean crack position is relevant, and the sub-grain roughness of the crack front can be safely ignored. The second option consists in coarse-graining the description of the crack and consider only effective parameters and scales which do not directly reflect the microstructure. After defining our notations, we will come back to this discussion.

The problem to solve here concerns a solid medium consisting of elastic brittle grains or potential arrest sites. The grain size or the site spacing is considered to be of extension $1 / \lambda$, with a randomly distributed toughness, characterized by a probability density function, $h\left(K_{c}\right)$, and no spatial correlations on scales larger than $1 / \lambda$ are assumed [28]. In contrast, the elastic properties of the grains are considered to be homogeneous. Therefore, for a given crack geometry, the stress intensity factor $K_{I}$ - assumed here to be the only relevant parameter - is supposed to be known as a function of the position of the crack tip $x$ and the remote stress field. This is a case that we will refer to as being two dimensional. Figure 1 shows schematically the notations used herein. Similar hypotheses have also been made to analyze the crack advance in fiber reinforced composites. However, instead of toughness distributions, strength distributions have been used [29,30,24,31].

In three dimensions, we need not only to account for the mean crack position, but also for the full crack front morphology. Even in the limit of a vanishing roughness of the crack front, the local stress intensity factor depends on the complete crack front shape through a long-range convolution kernel. This feature precludes any simple analytical approach to the problem. The crack front roughness adapts itself to the heterogeneity of the local grain toughness. A statistical model taking into account this long-range elastic interaction along the front has been proposed by Schmittbuhl et al. [25], and the statistics of the macroscopic loading necessary to propagate the crack has been studied in details by Skoe el al. for the same model [27]. It has been shown in particular that the macroscopic loading displays a singular behavior close to an effective depinning threshold, as a result of a mapping onto a genuine second-order phase transition. This allows one to define an effective or "equivalent" 
toughness that accounts for the roughening of the crack front. Consequently, the distribution of this effective toughness is not simply related to that of the individual grains. If one uses this effective toughness (or the maximum of it over some chosen propagation distance) and that this distance is designed to be larger than the roughness amplitude of the crack front, one comes back to the two-dimensional case. In that case however, the distance $1 / \lambda$ is no longer equal to the grain size, but rather to the roughness of the crack front. Similarly, the statistical distribution $h\left(K_{c}\right)$ is that of the effective toughness, and thus is not simply related to that of individual grains. We will not address in the present study the relation of this effective toughness to that of the grains, but rather use the simplified two dimensional picture, where the crack front is only parameterized by its mean position, and the effective distribution of critical toughness is assumed to be known (measured experimentally or obtained from numerical simulations). For the sake of simplicity, we will often refer to the scale $1 / \lambda$ as being equal to the size of one grain (as in $2 \mathrm{D}$ situation) even if it has a more involved definition in $3 \mathrm{D}$ cases.

For a given crack path, the stress intensity factor $K_{I}(x)$ is known as well as the toughness of each grain, $K_{c}(x)$. The latter field is a random function piecewise constant over a length $1 / \lambda$ and a stress intensity factor $K_{I}(x)$ is supposed to vary slowly over the grain size (i.e., $\lambda x \gg 1)$. The cases studied below are bi-dimensional, and cracks are considered to be straight (i.e., a transgranular fracture mode). One can note that this is not a restriction and intergranular fracture can be considered in the same framework [16]. The fluctuation of the toughness may be the result of random crystallographic orientations as observed in ceramic sintering [32] or arrest sites in glass (the term "site" will be used throughout the paper with this general meaning). The question addressed herein concerns the conditions for crack arrest to occur in a random microstructure for any time, either finite or infinite.

The case of a time-independent (i.e., instantaneous) crack propagation criterion is considered in the third section, and will constitute a basic result from which the time-dependent case will be derived. In the fourth section, the subcritical crack growth regime is specifically addressed. The analysis of the statistical distribution of time required for the failure of a 
single "grain" in the microstructure leads to the conclusion that the toughest site is the one that dominates over all other sites along a given crack path. This allows us to express the probability distribution in terms of Lévy stable laws $[33,34]$. Such a result relates the timedependent crack propagation probability law to a time-independent problem. An equivalent formulation, which consists in finding the length distribution at fixed time, is derived. A last section is devoted to the analysis of a case study of a thin walled tube loaded by a temperature differential.

\section{INSTANTANEOUS CRACK PROPAGATION AND ENDURANCE LIMITS}

Two limit cases are first analyzed. They correspond to instantaneous propagation conditions and endurance (i.e., for any finite or infinite time).

\section{A. Instantaneous Crack Propagation}

Subcritical crack growth is first ignored, and thus a simple Griffith criterion [35] is assumed to be applicable. A crack has reached an extension $x$ and is characterized by a stress intensity factor $K_{I}(x)$ known from the geometry of the crack path and the applied load. At position $x$, the crack tip encounters a potential arrest site characterized by a toughness $K_{c}(x)$. The standard Griffith criterion gives the following condition

$$
K_{c}(x)<K_{I}(x)
$$

for which the crack will propagate through the site at position $x$. By using the probability density for the toughness distribution, the probability of propagation through the site is denoted by $\rho(x)$

$$
\rho(x)=P\left(K_{c}(x)<K_{I}(x)\right)=\int_{K_{c}^{\min }}^{K_{I}(x)} h(K) \mathrm{d} K=H\left(K_{I}(x)\right),
$$

where $H$ is the cumulative distribution of toughnesses (i.e., the integral of $h$ ). Since the toughness is always positive, $K_{c}$ varies within the range $\left[K_{c}^{\min }, K_{c}^{\max }\right]$ with: $0 \leq K_{c}^{\min }$ and 
$K_{c}^{\max } \leq+\infty$. For instance, a beta distribution can be used to analyze microhardness experiments on $\mathrm{Al}_{2} \mathrm{O}_{3}$ ceramics for which $K_{c}^{\min }=0$ and $K_{c}^{\max }<+\infty$ [16]. By assuming no spatial correlation in the site toughness, the probability, $P(a, b)$, that a crack may start at a length $a$ and reach (at least) a length $b$ can be derived. For a given crack path, let us introduce the abscissa $x_{i}$ of each site $i$ for which the stress intensity factor is minimum, and write (because of the site-independence realization of the toughness)

$$
P(a, b)=\prod_{i=1}^{n} \rho\left(x_{i}\right)
$$

Since it is assumed that the site intersection with the crack path can be characterized by a unique length scale $1 / \lambda$, a continuous limit for the above probability when $\lambda(b-a) \gg 1$ is written as

$$
P(a, b)=\exp \left[\lambda \int_{a}^{b} \log \left[H\left(K_{I}(x)\right)\right] \mathrm{d} x\right] .
$$

This model is close to the one proposed by Jeulin [36] in which the microstructure is assumed to be described by a Poisson mosaic. A Poisson tessellation of parameter $\lambda$ defines the grain boundaries. The latter are made of Poisson lines in the plane for a two-dimensional medium. The hypotheses made herein (i.e., a constant grain size) will make the following derivations easier.

\section{B. Endurance}

If the instantaneous crack propagation condition, Eq. (1), is not satisfied, subcritical crack propagation may occur. In such a case, the crack length increases with time $[14,37]$ according to a modified crack propagation law

$$
\frac{d x}{d t}=C\left[\frac{\left\langle K_{I}(x)-K_{t h}(x)\right\rangle}{\left\langle K_{c}(x)-K_{I}(x)\right\rangle}\right]^{m}
$$

for $K_{t h}(x)<K_{I}(x)<K_{c}(x)$ where $K_{t h}$ is the threshold toughness below which no propagation occurs, $C$ a characteristic velocity, $m$ a sensitivity exponent, the two latter quantities being material (and environment) dependent constants, and $\langle$.$\rangle the Macauley brackets (i.e.,$ 
positive part of '.'). Such an empirical relation characterizes a regime when crack growth is no longer simply dictated by the mechanical loading, but is assisted by a (rate-limiting) chemical reaction, as in stress-induced corrosion $[38,13]$. We do not enter into the discussion of the complex mechanisms at play, and interfering effects such as limitation of the crack velocity by the diffusion of chemically active species (such as water) from the environment [12], or the existence of a non-zero threshold $K_{t h}$ [39]. Rather, this empirical law is accepted as ruling the crack propagation, and trusting numerous experimental studies which have validated such an algebraic form in different ranges of velocities. In section IV-D, the identification of the relevant parameters is discussed for a commonly encountered subcritical crack growth law.

If $K_{I}(x) \geq K_{c}(x)$, the crack length increases instantaneously, while if $K_{I}(x)<K_{t h}(x)$, no propagation occurs. In the following, a time-independent loading will be assumed (i.e., static fatigue). The instantaneous extension of the crack is thus described by the results of the previous section. In the opposite limit of infinite time, i.e., the endurance regime, a different, though similar, result is expected. The arrest criterion is no longer given by $K_{I}(x)<K_{c}(x)$, but rather $K_{I}(x)<K_{t h}(x)$. Since the mathematical form of this criterion is identical to that for instantaneous propagation, the previous approach is extended by substituting $K_{t h}$ for $K_{c}$.

For the sake of simplicity, $K_{t h}$ and $K_{c}$ are assumed to be proportional to each other, such as what would occur with anisotropic grains in ceramics where the disorientation would be responsible for an effective heterogeneity of toughness. The toughness ratio is introduced

$$
\mu=\frac{K_{t h}(x)}{K_{c}(x)}
$$

and is considered to be identical for any value of the toughness. Therefore the statistical distribution $h$ characterizes both the upper $K_{c}$ and lower limit $K_{t h}$ toughnesses of the subcritical regime. The probability that a crack grow from $a$ to $b$ after an infinite time is thus simply deduced from Eqs. (4) and (6)

$$
P(a, b, T \rightarrow+\infty)=\exp \left[\lambda \int_{a}^{b} \log \left[H\left(K_{I}(x) / \mu\right)\right] \mathrm{d} x\right] .
$$


After having written the two limits of a crack extension, i.e., instantaneous propagation, Eq. (4), and endurance, Eq. (7), the condition for a propagation during a time $t$ less than a given value $T$ is now considered.

\section{SUBCRITICAL CRACK PROPAGATION}

The crack propagation law, Eq. (5), allows us to compute the time $\tau$ for a crack to propagate through a grain of length $1 / \lambda$ or between two sites $1 / \lambda$ apart (small as compared to the current size $x$ )

$$
\tau=\frac{1}{\lambda C}\left(\frac{\left\langle K_{c}-K_{I}\right\rangle}{\left\langle K_{I}-\mu K_{c}\right\rangle}\right)^{m},
$$

where $K_{I}$ is the value of the stress intensity factor at the considered site. One can note that $\tau$ is a random variable ranging from 0 to $+\infty$, with a probability density function using the same parameters as the toughness distribution and the material-dependent constants $C, m$, $\lambda$ and $\mu$. The probability for a crack to propagate along a given crack path $[a, b]$ during a time $t$ less than a given value $T$ is

$$
P(a, b, T)=P\left(\sum_{i=1}^{\lambda(b-a)} \tau_{i}<T\right),
$$

where $\tau_{i}$ is the time used by the crack to propagate through site $i$ of the given crack path, and $\lambda(b-a)$ the number of traversed sites by the crack.

This expression shows the difficulty that arises at finite (non-zero) times. One can no longer use a purely local criterion. Rather, a long time may be spent for one site if elsewhere along the crack path the velocity is large enough to compensate. This gives a non-local condition that appears more difficult to account for than the previous limits $T=0$ (Eq. (4)) or $T=+\infty$ (Eq. (7)).

Yet, the sum of uncorrelated random variables has received a lot of attention because of its ubiquitous character in many statistical problems. In particular, the central limit theorem is a very powerful tool to handle such problems. However, the latter theorem only 
holds for sums of random variables having a finite variance. In the following, it will be shown that this is not the case here. Extensions of this theorem are needed, and unexpectedly, this will yield a very simplified picture of the problem, close to the two previous limit cases.

\section{A. Asymptotic Properties of the Random Variable $\tau$}

For large values of $T$, an approximation is determined for the probability $P(\tau>T)$ that the time required to traverse a site exceed $T$. A large time means that $K_{I}$ is close to $\mu K_{c}=K_{t h}$. Let us introduce the small and positive parameter $\epsilon$ such that

$$
\frac{K_{c}}{K_{I}}=\frac{1}{\mu}-\epsilon
$$

By using the crack growth law (5), time $T$ can be expressed as a function of $\epsilon$,

$$
\begin{aligned}
T & =\frac{1}{\lambda C}\left(\frac{1-\mu-\mu \epsilon}{\mu^{2} \epsilon}\right)^{m} \\
& \approx \frac{1}{\lambda C}\left(\frac{1-\mu}{\mu^{2}}\right)^{m} \epsilon^{-m} .
\end{aligned}
$$

The probability that the time to go through one site exceed $T$ can be divided into two parts, first the probability that the site will resist forever, i.e., $K_{t h}>K_{I}$, and the probability that the time remains finite but larger than $T$. The latter probability, denoted by $P(T<\tau<$ $+\infty)$, is such that

$$
\frac{1}{\mu}-\epsilon(T)<\frac{K_{c}}{K_{I}}<\frac{1}{\mu}
$$

For large enough $T$ (i.e., small enough $\epsilon$ ), this probability can be approximated by

$$
P(T<\tau<+\infty)=\int_{K_{I}(1 / \mu-\epsilon)}^{K_{I} / \mu} h(K) \mathrm{d} K \approx K_{I} h\left(K_{I} / \mu\right) \epsilon
$$

provided $h\left(K_{I} / \mu\right)$ is non-zero. Therefore, the following asymptotic form for the distribution of time is

$$
P(T<\tau<+\infty) \approx K_{I} h\left(K_{I} / \mu\right)\left(\frac{\Theta}{T}\right)^{1 / m}
$$


where

$$
\Theta=\left(\frac{1-\mu}{\mu^{2}}\right)^{m} \frac{1}{\lambda C}
$$

The important result is that, asymptotically, $P$ decays algebraically to zero for large $T$ as a power-law with a low exponent (e.g., for alumina, $1 / m$ is of the order of $1 / 7$ ). In particular for $m>1 / 2$ the variance of $T$ diverges, and for $m>1$ even the first moment of the distribution, i.e., the mean propagation time, diverges. Furthermore, $\Theta$ scales as $1 / \lambda C$ as imposed from dimensional analysis. However, the dimensionless prefactor may easily induce a strong underestimation of this characteristic time, e.g., for $\mu=0.5$ and $m=7$ to consider reasonable values, a prefactor of the order of $10^{2}$ is found.

\section{B. Probability of Sub-critical Crack Propagation}

In the beginning of this section, we have seen that the probability for a crack to go from $a$ to $b$ in a time less than $T$ involves a condition on the sum $S_{n}$ of $n$ propagation times $\tau_{i}$ : $S_{n}=\sum_{i=1}^{n} \tau_{i}$. As shown above, $P(\tau)$ has a power-law tail $\tau^{-\alpha}$ with an exponent $\alpha=1 / m$, and thus quite generally the variance of the times is infinite. Consequently, one cannot use the central limit theorem [40] for this sum. Lévy [33,34] has proven that such a sum (when rescaled by $n^{1 / \alpha}$ ) converges in law towards a stable form, called a Lévy distribution, characterized by the exponent $\alpha$ (in the general case, an additional asymmetry parameter is required, but in our case where $\tau$ is always positive, one has to consider the one-sided family of Lévy distribution completely characterized by $\alpha$ ). The sum $S_{n}$ will converge for large $n$ towards $n^{1 / \alpha} \mathcal{L}_{\alpha}\left(S_{n}^{1 / \alpha}\right)$. The interested reader is referred to Lévy [34] for a detailed presentation of $\mathcal{L}$. Let us simply recall the most important features:

- $\mathcal{L}$ has the same algebraic behavior for large arguments as each of the distribution of individual times,

- if $M_{n}$ denotes the maximum of the series of $n$ random numbers $\tau_{i}$, 


$$
M_{n}=\max _{i=1}^{n} \tau_{i}
$$

then both $S_{n}$ and $M_{n}$ will scale as $n^{1 / \alpha}$, and their ratio is well-behaved and approaches a finite value for $\alpha<1[41]$

$$
\lim _{n \rightarrow \infty}\left(\frac{S_{n}}{M_{n}}\right)=\frac{1}{1-\alpha}
$$

where the ratio is to be understood as the expectation value.

By considering a typical value of $m=7$ (see next section), or $\alpha=1 / 7$, one can see that, on average, one single time provides $86 \%$ of the sum. Therefore, albeit all sites contribute to the total time, the very wide distribution of these times is such that the sum is very close to the value of the maximum. For the sum $S_{n}$ to be less than a prescribed time $T$, it is approximately equivalent to require that the maximum $M_{n}$ be less than $(1-\alpha) T$. Thus, one encounters a problem which is very close to the two limits analyzed in the previous section.

One simply needs to compute the value of $K_{c}$ such that the corresponding time amounts to $(1-1 / m) T$. A simple inversion yields

$$
K_{c}=K_{I} \Phi(T)
$$

where

$$
\Phi(T)=\frac{1+[(1-1 / m) \lambda C T]^{1 / m}}{1+\mu[(1-1 / m) \lambda C T]^{1 / m}} .
$$

One may further note that the small correction due to the difference between the sum and the maximum local time comes into play through the factor $(1-1 / m)^{1 / m}$ which, for large $m$, can be approximated by $1-1 / m^{2}$. For $m=7$, the latter quantity differs from 1 by only $2 \%$. Therefore, up to second order terms in $1 / m$, one may simplify the above expression and rather use

$$
\Phi(T) \approx \frac{1+[\lambda C T]^{1 / m}}{1+\mu[\lambda C T]^{1 / m}}
$$


By simply borrowing from the first section the expression for the probability for the crack to propagate from $a$ to $b$ and using a time-dependent correction for $K_{I}$ given by the function $\Phi(T)$ defined in Eq. (19), the propagation probability $P(a, b, T)$ can be written as

$$
P(a, b, T)=\exp \left[\lambda \int_{a}^{b} \log \left[H\left(K_{I}(x) \Phi(T)\right)\right] \mathrm{d} x\right] .
$$

Equation (21) constitutes the main result of the paper. It allows for the computation of the propagation probability by simply correcting (i.e., multiplying) the stress intensity factor by the function $\Phi$ defined in Eqs. (19) or (20).

\section{Time and Crack Length Distributions}

From Eq. (21), two main distributions can be derived. The probability density $p_{a, b}$ that the crack starting from $a$ reaches $b$ between times $T$ and $T+d T$ is

$$
p_{a, b}(T)=\frac{\partial P(a, b, T)}{\partial T}=\lambda P(a, b, T) \frac{\mathrm{d} \Phi(T)}{\mathrm{d} T} \int_{a}^{b} \frac{h\left[K_{I}(x) \Phi(T)\right] K_{I}(x)}{H\left[K_{I}(x) \Phi(T)\right]} \mathrm{d} x
$$

The probability density $p_{a, b}$ is therefore proportional to $\mathrm{d} \Phi(T) / \mathrm{d} T$, itself directly related to the subcritical crack propagation law.

Up to now, we have considered the time evolution of the probability distribution for a crack to grow along a prescribed crack path. Alternatively, one may be interested in the crack length distribution at fixed time. No new derivation is needed. Let us introduce the notation $x(t)$ for the position of the crack tip as a function of time, and $t(x)$ the inverse function, the function $x(t)$ being bijective. The two following events are equivalent

$$
\{x(T)>b\}=\{t(b)<T\} .
$$

Equation (22) gave the expression of the probability for the second event. Hence, it also gives the probability for the first one. At time $T$, the probability density $p_{a, T}$ that the crack tip lie in between $b$ and $b+\mathrm{d} b$ is

$$
p_{a, T}(b)=\frac{\partial P(a, b, T)}{\partial b}=\lambda P(a, b, T) \log \left[H\left[K_{I}(b) \Phi(T)\right]\right]
$$


This last result only involves the (multiplicative) correction by the function $\Phi$ of the applied stress intensity factor.

\section{Time-Correction Function}

The previous derivations led us to introduce the function $\Phi$, which will be referred to as the time-correction function. Figure 2 shows the change of $\Phi$ with the dimensionless time $\lambda C T$. It can be seen that the approximation of Eq. (19) by Eq. (20) is very close for the two higher values of $m$. For the lowest value, a downward shift of the approximation with respect to the actual function can be seen. However, the general trend is the same and as a first approximation, Eq. (20) will be used: a skeletal point is obtained for the value $\lambda C T=1$ for which $\Phi(1)=2 /(\mu+1)$ for any value of the sensitivity exponent $m$. It can be noted that the value of the ratio $\mu$ is not independent of the usual crack propagation parameters $A$ and $p$ of an Evans-Wiederhorn propagation law [14,15,37]

$$
\frac{d x}{d t}=A\left(\frac{K_{I}}{K_{c 0}}\right)^{p}
$$

where $K_{c 0}$ is a characteristic (e.g., macroscopic) toughness. The threshold stress intensity factor can be determined by setting the value of $K_{I}\left(d x / d t=V_{0}\right)=\mu K_{c 0}$ so that

$$
\mu=\left(\frac{V_{0}}{A}\right)^{1 / p}
$$

The last two parameters $C$ and $m$ are determined by assuming that the propagation velocity and its first derivative are identical for the two subcritical propagation models when $K_{I} / K_{c 0}=(1+\mu) / 2$, i.e., the skeletal point of the function $\Phi$

$$
C=A\left(\frac{1+\mu}{2}\right)^{p} \quad \text { and } \quad m=\frac{p(1-\mu)}{2(1+\mu)} .
$$

Figure 3 shows the use of the present procedure to determine the relevant constants for a $99.6 \%$ alumina ceramic whose original parameters were determined by using flexural experiments performed at different stress rates [42]: $K_{c 0}=4.5 \mathrm{MPa} \sqrt{\mathrm{m}}, A=6.9 \mathrm{~m} / \mathrm{s}$ and

$p=52$. The corresponding parameters are given as follows: $\mu=0.57$ (when $V_{0}=10^{-12}$ 
$\mathrm{m} / \mathrm{s}), C=2.1 \times 10^{-5} \mathrm{~m} / \mathrm{s}$ and $m=7.2$. The results derived so far are now used to study crack propagation in a thin walled tube subjected to a temperature gradient.

\section{THERMOELASTIC LOADING OF A THIN WALLED TUBE}

A thin walled tube of radius $R$ and thickness $h$ (Fig. 4) is subjected to a variable temperature differential between the inner and outer surface between 0 and $\Delta \theta$. If $(z, \varphi)$ are coordinates along the length and circumference of the cylinder, the thermoelastic solution is $(h / R \ll 1)$

$$
\sigma_{z z}^{\theta}=\sigma_{\varphi \varphi}^{\theta}=\sigma^{\theta} \frac{2 x}{h}
$$

with

$$
\sigma^{\theta}=\frac{E \alpha \Delta \theta}{2(1-\nu)}
$$

where E, $\nu$ are the Young's modulus and Poisson's ratio of the considered material, respectively, $\alpha$ the coefficient of thermal expansion, $x=0$ the mid-surface of the tube wall, $x=h / 2$ the outer surface and $x=-h / 2$ the inner surface. This setting corresponds to the so-called Bree problem [43] used in the shakedown analysis for elastoplastic materials [44]. In the present case, one is interested in studying the propagation of cracks from initial defects caused by the residual stresses induced by the (constant) temperature gradient through the wall thickness. The onset of crack propagation from initial defects will be referred to as inception as opposed to initiation for which a crack is formed during the load history. From a deterministic perspective, inception occurs on the outer surface when $\Delta \theta>0$. Conversely, when $\Delta \theta<0$, inception occurs on the inner surface. It is worth noting that, even though the propagation condition is not described by the weakest link assumption, inception is still assumed to be. Consequently, inception may be modelled by a Weibull law [45] and the location of inception becomes random. Following $\mathrm{Oh}$ and Finnie [46], one can derive the 
distribution of inception sites within the thickness of the tube for any direction in the $(z, \varphi)$ frame since the stress is equibiaxial. In particular, the average inception height, $\bar{h}$, is given by $(\Delta \theta>0)$

$$
\bar{h}=\int_{0}^{+\infty} \int_{0}^{h / 2} x \psi\left(\sigma^{t}, x\right) \mathrm{d} x \mathrm{~d} \sigma^{t}
$$

with

$$
\psi\left(\sigma^{t}, x\right)=\frac{1-P_{I}\left(\sigma^{t}\right)}{L_{0}} \frac{\partial}{\partial \sigma^{t}}\left(\frac{\sigma\left(\sigma^{t}, x\right)}{S_{0}}\right)^{M}
$$

and

$$
P_{I}\left(\sigma^{\theta}\right)=\int_{0}^{\sigma^{\theta}} \int_{0}^{h / 2} \psi\left(\sigma^{t}, x\right) \mathrm{d} x \mathrm{~d} \sigma^{t}
$$

where $P_{I}$ is the inception probability, $M$ the Weibull modulus, $S_{0}$ the scale parameter and $L_{0}$ the corresponding gauge length. No special care has to be taken in deriving Eq. (30), since the integration domain is independent of the applied load [47]. If one considers only the formation of longitudinal cracks (induced by the hoop stresses $\sigma_{\varphi \varphi}$ ), the average inception height becomes

$$
\bar{h}=\frac{h}{2} \frac{M+1}{M+2}
$$

Equation (33) shows that the likely inception sites are located near the external surface. The higher $M$, the closer $\bar{h}$ to the external surface. In the limiting case of a deterministic and local failure strength (i.e., $M \rightarrow+\infty$ ), it is found, as expected, that the inception site is located on the outer surface.

In the following, it is assumed that inception will occur on the outer surface from a defect of size of the order of the size of the microstructure (i.e., $1 / \lambda)$. If the defect is modelled by a semi-circular crack of radius $1 / \lambda \ll h$ (Fig. 5), the stress intensity factor $K$ along the radius of the crack can be written as

$$
K(\beta)=Y(\beta) \frac{\sigma^{\theta}}{\sqrt{\lambda}},
$$


where $Y$ is a dimensionless shape factor dependent on the defect type and the considered location $\beta$. Solutions are available when $10^{\circ}<\beta<170^{\circ}[48]$. Near the outer surface, a 'vertex' singularity is observed. A lower bound $K_{\min }$ to the stress intensity factor is reached when $\beta=90^{\circ}$

$$
K_{\min }=1.16 \frac{\sigma^{\theta}}{\sqrt{\lambda}}
$$

Propagation is therefore likely to occur in the longitudinal direction rather than through the thickness. This fact is even more likely in the present situation because of the decrease of the stress field through the thickness. Consequently, a channelling crack will develop in a similar way as in pre-tensioned films [49]. At each stage of the growth, the channel front adjusts itself to a curved shape, such that the energy release rate at every point of the front is identical. By construction, this problem is three-dimensional and an accurate solution would require involved computations. Yet, after the crack length exceeds a few times the initial radius $1 / \lambda$, a steady state configuration is reached: the front maintains its shape as it propagates, and the energy released per unit advance no longer depends on the initial geometry, nor on the channel length. The energy release rate can be evaluated without knowing the shape of the front by using two plane problems. By subtracting the strain energy stored in a unit strip far behind of the front, from that far ahead, one can derive different formulas [49]. One of them is

$$
\mathcal{G}_{s s}=\lambda \int_{0}^{1 / \lambda} G(a) \mathrm{d} a
$$

where $\mathcal{G}_{\text {ss }}$ denotes the steady state energy release rate, $\mathcal{G}(a)$ the energy release rate of a plane strain crack of depth $a$. When $\lambda h \gg 1$, an approximation is given by an edge crack in a semi-infinite plane. The corresponding stress intensity factor is given by $K(a)=1.12 \sigma^{\theta} \sqrt{\pi a}$ [48] so that the steady state stress intensity factor $K_{s s}$ can be calculated by using Eq. (36)

$$
K_{s s}=1.41 \frac{\sigma^{\theta}}{\sqrt{\lambda}}
$$

As expected, $K_{s s}$ is independent of the current crack length, it only depends upon the initial height of the crack. It can be noted that a tunnelling crack [50] can also be used to analyze 
the propagation condition of a crack whose propagation inception occurs within the bulk and when the crack does not intersect the inner or outer surface (e.g., the propagation is parallel to the $z$-direction). Under this assumption, a steady state configuration is also reached and the corresponding stress intensity factor now depends on the location $x(1<\lambda x<(\lambda h) / 2-1)$

$$
K_{s s}(x)=0.89 \frac{\sigma^{\theta}}{\sqrt{\lambda}} \frac{2 x}{h} .
$$

By comparing Eqs. (37) and (38), it can be concluded that channelling cracks are more likely to occur than tunnelling cracks near the outer surface. Furthermore, the crack front is likely to reach very quickly the outer surface anyway.

The channelling crack inducing a steady state situation (with a constant stress intensity factor $K_{s s}$ ), the propagation conditions are easy to analyze. Furthermore, since the crack height is equal to $1 / \lambda$, the $2 \mathrm{D}$ propagation model derived herein can be used. The crack extension probability $P(a, b, T)$ can be written as

$$
P(a, b, T)=H\left[K_{s s} \Phi(T)\right]^{\lambda(b-a)}
$$

where $\lambda(b-a)$ is the number of traversed grains. The toughness $K_{c}$ is here assumed to be bounded by $K_{c}^{\min }$ and $K_{c}^{\max }$ so that: $0 \leq K_{c}^{\min } \leq K_{c} \leq K_{c}^{\max }<+\infty$. Equation (39) shows that, depending on the level of $K_{s s}$ and the considered time $T$, three different regimes are obtained (Fig. 6). First, a deterministic propagation regime when $K_{s s} \Phi(T)>K_{c}^{\max }$ in which the crack will traverse the whole tube length. Second, a deterministic arrest regime when $K_{s s} \Phi(T)<K_{c}^{\mathrm{min}}$ in which the crack is arrested. Finally, a third intermediate situation, referred to as probabilistic propagation/arrest regime, when $K_{c}^{\min } \leq K_{s s} \Phi(T) \leq K_{c}^{\max }$. In the latter regime, the longer the crack, the more likely crack arrest. This result is due to the random distribution of toughnesses and the fact that when the crack propagates, it is more likely to hit an arrest site (i.e., a grain with a high toughness). Therefore the propagation probability decreases as the propagation length increases. The curves plotted in Fig. 6 are upper estimates of the propagation conditions since the steady state stress intensity factor is on average greater than the inception stress intensity factor. However, for design purposes, 
the steady state condition is a good estimate. Finally, for small values of $\lambda(b-a)$, the discrete nature of the propagation is clearly visible. As soon as a grain boundary is traversed, there is a sudden drop of the propagation probability, since a new possible arrest site may be hit. Conversely, for larger values of $\lambda(b-a)$, typically 50 grains in the present case, the effect of the discreteness of the microstructure on the propagation can no longer be observed.

\section{CONCLUSION}

A model for crack propagation in elastic brittle media has been developed. It is based upon a distribution of random toughnesses from site to site suited either for a two dimensional geometry or a three-dimensional one provided that in the latter case, the grain scale and the statistical distribution of local toughness are reinterpreted as effective parameters defined on a scale larger that the natural crack front roughness. A general formulation has been derived for the propagation probability $P(a, b, T)$

$$
P(a, b, T)=\exp \left[\lambda \int_{a}^{b} \log \left[H\left(K_{I}(x) \Phi(T)\right)\right] \mathrm{d} x\right] .
$$

After having determined the instantaneous crack propagation probability (i.e., $\Phi(T=0)=$ 1) and the endurance crack propagation probability (i.e., $\Phi(T \rightarrow+\infty)=1 / \mu$ ), timedependent function $\Phi(T)$ has been obtained. The latter is only dependent upon the parameters modeling subcritical crack propagation in addition to the discretization $\lambda$.

The parameters of the propagation model are therefore restricted to the toughness probability density function that can be determined by using microhardness experiments and the subcritical crack propagation parameters. It can be noted that the model can be extended to incorporate the fact that the initial crack size $a$ can be random (e.g., a common assumption in models based upon the weakest link hypothesis). The discretization parameter $\lambda$ needs to be further studied to get a good evaluation. It is proposed that subcritical crack propagation constitutes yet another way of tuning $\lambda$.

The case study dealing with propagation of cracks in a thin walled tube submitted to a temperature gradient through its thickness showed that a steady state propagation can be 
reached. It also allowed for the determination of three different regimes. Two deterministic regimes, namely one for which propagation is certain to occur, and one for which arrest always happens. In between, a probabilistic regime is found for which the longer the crack, the smaller the crack extension probability. This result is typical of the strongest link assumption where the propagation event is dictated by the strongest event (i.e., the toughest grain on the propagation path).

Finally, one can note that, even though static fatigue was considered herein, the same results can be directly applied in cyclic fatigue (i.e., time $T$ becomes equal to the number of cycles $N$ in Eq. (40)) when the stress amplitude is constant with the number of cycles $N$. Instead of an Evans-Wiederhorn crack propagation law, a Paris' law [51] would be preferred or any modification accounting for near threshold behavior induced by crack closure [52-54]. 


\section{REFERENCES}

[1] Freudenthal, A.M., 1968, "Statistical Approach to Brittle Fracture," in: Liebowitz, H., ed., Fracture, Academic Press, New York, USA, 2, pp. 591-619.

[2] Weibull, W., 1939, "A Statistical Theory of the Strength of Materials," Roy. Swed. Inst. Eng. Res., 151.

[3] Weibull, W., 1951, "A Statistical Distribution Function of Wide Applicability," ASME J. Appl. Mech., 18, pp. 293-297.

[4] Jayatilaka, A. de S., and Trustrum, K., 1977, "Statistical Approach to Brittle Fracture," J. Mater. Sci., 12, pp. 1426-1430.

[5] Munz, D., and Fett, T., 1999, "Scatter of Mechanical Properties," in: Ceramics, Springer, Berlin, Germany, pp. 137-158.

[6] Batdorf, S.B., and Heinisch Jr., H.L., 1978, "Weakest Link Theory Reformulated for Arbitrary Fracture Criterion," J. Am. Ceram. Soc. 61, 355-358.

[7] Evans, A.G., 1978, "A General Approach for the Statistical Analysis of Multiaxial Fracture," J. Am. Ceram. Soc., 61, pp. 302-308.

[8] Davies, D.G.S., 1973, "The Statistical Approach to Engineering Design in Ceramics," Proc. Brit. Ceram. Soc., 22, pp. 429-452.

[9] Aveston, J., Cooper, G.A., and Kelly, A., 1971, "Single and Multiple Fracture," Proc. National Physical Laboratory: Properties of Fiber Composites, IPC Science and Technology Press, Surrey, UK, pp. 15-26.

[10] Riou, P., Denoual, C., and Cottenot, C.E., 1998, "Visualization of the Damage Evolution in Impacted Silicon Carbide Ceramics," Int. J. Impact Eng., 21, pp. 225-235.

[11] Denoual C., and Hild F., 2000, "A Damage Model for the Dynamic Fragmentation of Brittle Solids," Comp. Meth. Appl. Mech. Eng., 183, pp. 247-258. 
[12] Wiederhorn, S.M., 1967, "Influence of Water Vapor on Crack Propagation in SodaLime-Silicate Glass," J. Am. Ceram. Soc., 50, pp. 407-417.

[13] Wiederhorn, S.M., and Bolz, L.H., 1970, "Stress Corrosion and Static Fatigue of Glass," J. Am. Ceram. Soc., 56, 192-197.

[14] Evans, A.G., 1972, "A Method for Evaluating the Time-Dependent Failure Characteristics of Brittle Materials - and its Application to Polycrystalline Alumina," J. Mater. Sci., 7, pp. 1137-1146.

[15] Evans, A.G., and Wiederhorn, S.M., 1974, "Crack Propagation and Failure Prediction in Silicon Nitride at Elevated Temperature," J. Mater. Sci., 9, pp. 270-278.

[16] Charles, Y., and Hild, F., 2002, "Crack Arrest in Ceramic/Steel Assemblies," Int. J. Fract., 15(3), pp. 251-272.

[17] Davidge, R.W., McLaren, J.R., and Tappin, G., 1973, "Strength-Probability-Time (SPT) Relationship in Ceramics," J. Mater. Sci., 8, pp. 1699-1705.

[18] Evans, A.G., and Wiederhorn, S.M., 1974, "Proof Testing of Ceramic Materials-An Analytical Basis for Failure Prediction," Int. J. Fract., 10, pp. 379-392.

[19] Evans, A.G., 1980, "Fatigue in Ceramics," Int. J. Fract., 16(5), pp. 485-498.

[20] Brinkman, C.R., and Duffy, S.F., eds., 1994, Life Prediction Methodologies and Data for Ceramic Materials, ASTM STP 1211, Philadelphia, USA.

[21] Bower, A.F., and Ortiz, M., 1991, "A Three-Dimensional Analysis of Crack Trapping and Bridging by Tough Particles," J. Mech. Phys. Solids, 28(5), pp. 815-858.

[22] Bower, A.F., and Ortiz, M., 1993, "The Influence of Grain Size on the Toughness of Monolithic Ceramics," ASME J. Eng. Mat. Technol., 115, pp. 228-236.

[23] Curtin, W.A., 1997, "Toughening in Disordered Brittle Materials," Phys. Rev. B, 55, pp. $11270-11276$. 
[24] Curtin, W.A., 1998, "Stochastic Damage Evolution and Failure in Fiber-reinforced Composites," in Adv. Appl. Mech., 36, pp. 164.

[25] Schmittbuhl, J., Roux, S., Vilotte, J.-P., and Måløy, K.J., 1995, "Pinning of interfacial crack: Effect of non-local interactions," Phys. Rev. Lett. 74, pp. 1787-1790.

[26] Ramanathan, S., and D.S. Fisher, 1998, "Onset of Propagation of Planar Cracks in Heterogeneous Media," Phys. Rev. B, 58, pp. 6026-6046.

[27] Skoe, R., Vandembroucq, D., and Roux, S., 2002, "Front Propagation in Random Media: From Extremal to Activated Dynamics," to appear in Int. J. Modern Physics C.

[28] Chudnovsky, A., and Kunin, B., 1987, "A Probabilistic Model of Brittle Crack Formation," J. Appl. Phys., 62, pp. 4124-4129.

[29] Beyerlein, I.J., Phoenix, S.L., 1997, "Statistics of fracture for an elastic notched composite lamina containing Weibull fibers - Part I. Features from Monte-Carlo simulation," Eng. Fract. Mech., 57, pp. 241- 265.

[30] Beyerlein, I.J., Phoenix, S.L., 1997, "Statistics of fracture for an elastic notched composite lamina containing Weibull fibers - Part II. Probability models of crack growth," Eng. Fract. Mech. 57, pp. 267- 299.

[31] Landis, C.M., Beyerlein, I.J., and McMeeking, R.M., 2000, "Micromechanical Simulation of the Failure of Fiber Reinforced Composites", J. Mech. Phys. Solids, 48, pp. 621648.

[32] Kingery, W.D., Bowen, H.K., and Uhlmann, D.R., 1976, Introduction to Ceramics, John Wiley and Sons, Inc., New York, USA.

[33] Lévy, P., 1924, "Théorie des erreurs. La loi de Gauss et les lois exceptionnelles," Bull. Math. Soc., 52, pp. 49-85.

[34] Lévy, P., 1954, Théorie de l'addition des variables aléatoires, Gauthier-Villars, Paris 
(France).

[35] Griffith, A.A., 1921, "The Phenomenon of Rupture and Flow in Solids," Phil. Trans. Roy. Soc. London A221, pp. 163-197.

[36] Jeulin, D., 1994, "Fracture Statistics Models and Crack Propagation in Random Media," Appl. Mech. Rev., 47, pp. 141-150.

[37] Wiederhorn, S.M., 1974, "Subcritical Crack Growth in Ceramics," in: Brandt, R.C., Hasselman, D.P.H., Lange, F.F., eds., Fracture Mechanics of Ceramics, Plenum Press, New York, USA, pp. 613-646.

[38] Hillig, W.B., and Charles, R.J., 1965, "Surfaces, Stress-Dependent Surface Reactions, and Strength," in: Zackay, V.F., ed., High Strength Materials, John Wiley and Sons, Inc., New York, USA, pp. 682-705.

[39] Wan, K.-T., Lathabai, S., and Lawn, B.R., 1990, "Crack Velocity Functions and Thresholds in Brittle Materials," J. Eur. Ceram. Soc., 6, pp. 259-268.

[40] Lindeberg, J.W., 1922, "Eine neue Herleitung des Exponentialgesetzes in der Wahrscheinlichkeitsrechnung," Math. Zeitschrifft, 15, pp. 211-225.

[41] Feller, W., 1966, An Introduction to Probability Theory and its Applications, John Wiley and Sons, Inc., New York, USA.

[42] Fett, T., and Munz, D., 1994, "Lifetime Predictions of Ceramic Materials under Constant and Cyclic Load," in: Brinkman, C.R., Duffy, S.F., eds., Life Prediction Methodologies and Data for Ceramic Materials, ASTM STP 1211, Philadelphia (USA), pp. 161-174.

[43] Bree, I., 1967, "Elastic-Plastic Behaviour of Thin Tubes Subjected to Internal Pressure and Intermittent High Heat Fluxes with Applications to Fast-Nuclear-Reactor Fuel Elements," J. Strain Analysis, 2, pp. 226-238. 
[44] Megahed, M.M., Morrison, C.J., and Ponter, A.R.S., 1982, "A Theoretical and Experimental Investigation of Material Rachetting Rates in a Bree Beam Element," Int. J. Mech. Sci., 25, pp. 917-933.

[45] Hild, F., and Roux, S., 1991, "Fatigue Initiation in Heterogeneous Brittle Materials," Mech. Res. Comm., 18, pp. 409-414.

[46] Oh, H.L., and Finnie, I., 1970, "On the Location of Fracture in Brittle Solids-I Due to Static Loading," Int. J. Fract. Mech., 6, pp. 287-300.

[47] Hild, F., 1994, "On the Average Pull-Out Length of Fiber-Reinforced Composites," C. R. Acad. Sci. Paris, t. 319 (Série II), pp. 1123-1128.

[48] Tada, H., Paris, P.C., and Irwin, G.R., 1985, "The Stress Analysis of Cracks Handbook," Del Research, St Louis, USA.

[49] Hutchinson, J.W., and Suo, Z., 1992. "Mixed Mode Cracking in Layered Materials," in: Hutchinson, J.W., and Wu, T.Y., eds., Advances in Applied Mechanics, Academic Press, Inc., New York, USA, pp. 63-191.

[50] Suo, Z., 1990, "Failure of Brittle Adhesive Joints," Appl. Mech. Rev., 43, pp. S276-S279.

[51] Paris, P.C., Gomez, M.P., and Anderson, W.P., 1961, "A Critical Analysis of Crack Propagation Laws," The Trend in Engineering, 13, pp. 9-14.

[52] Elber, W., 1970, "Fatigue Crack Closure under Cyclic Tension," Eng. Fract. Mech., 2, pp. $37-45$.

[53] Elber, W., 1971, "The Significance of Fatigue Crack Closure," Damage Tolerance in Aircraft Structures, ASTM STP 486, Philadelphia, USA, pp. 230-242.

[54] Pellas, J., Baudin, G., and Robert, M., 1977, "Mesure et calcul du seuil de fissuration après surcharge," Recherche aérospatiale, 3, pp. 191-201. 


\section{List of Figures}

1 Propagation path from an initial length $a$ to a final length $b$ intersecting sites of spacing $1 / \lambda$ and random toughness. . . . . . . . . . . 27

2 Time-correction function $\Phi$ vs. dimensionless time $\lambda C T$ for different values of the sensitivity exponent $m$ when $\mu=0.6 \ldots \ldots \ldots . \ldots 28$

3 Identification of the crack propagation parameters $\mu, C, m$ from conventional Evans-Wiederhorn parameters $A, p$ for a $99.6 \%$ alumina ceramic for a conventional value of $V_{0} \ldots \ldots \ldots \ldots \ldots \ldots \ldots$

4 Thin-walled tube of average radius $R$ and thickness $h$. Frame of a volume element of the tube and distribution of stresses $\sigma_{z z}$ and $\sigma_{\varphi \varphi}$ through the

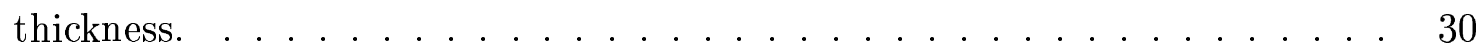

5 Longitudinal crack initiated on the outer surface of the tube. Initial semicircular crack and channelling crack experiencing a steady-state propagation.

6 Crack extension probability vs. dimensionless extension length for different values of the cumulative probability $H\left[K_{s s} \Phi(T)\right]$. Three different regimes can be observed. . . . . . . . . . . . . . . . . 32 


\section{FIGURES}

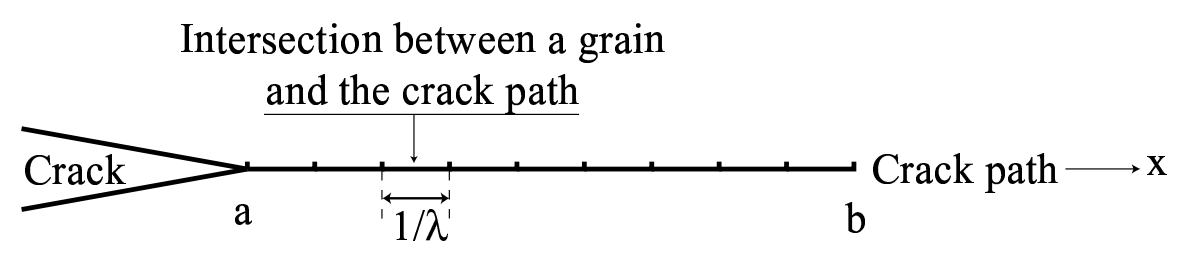

FIG. 1. Propagation path from an initial length $a$ to a final length $b$ intersecting sites of spacing $1 / \lambda$ and random toughness.

Charles et al. 


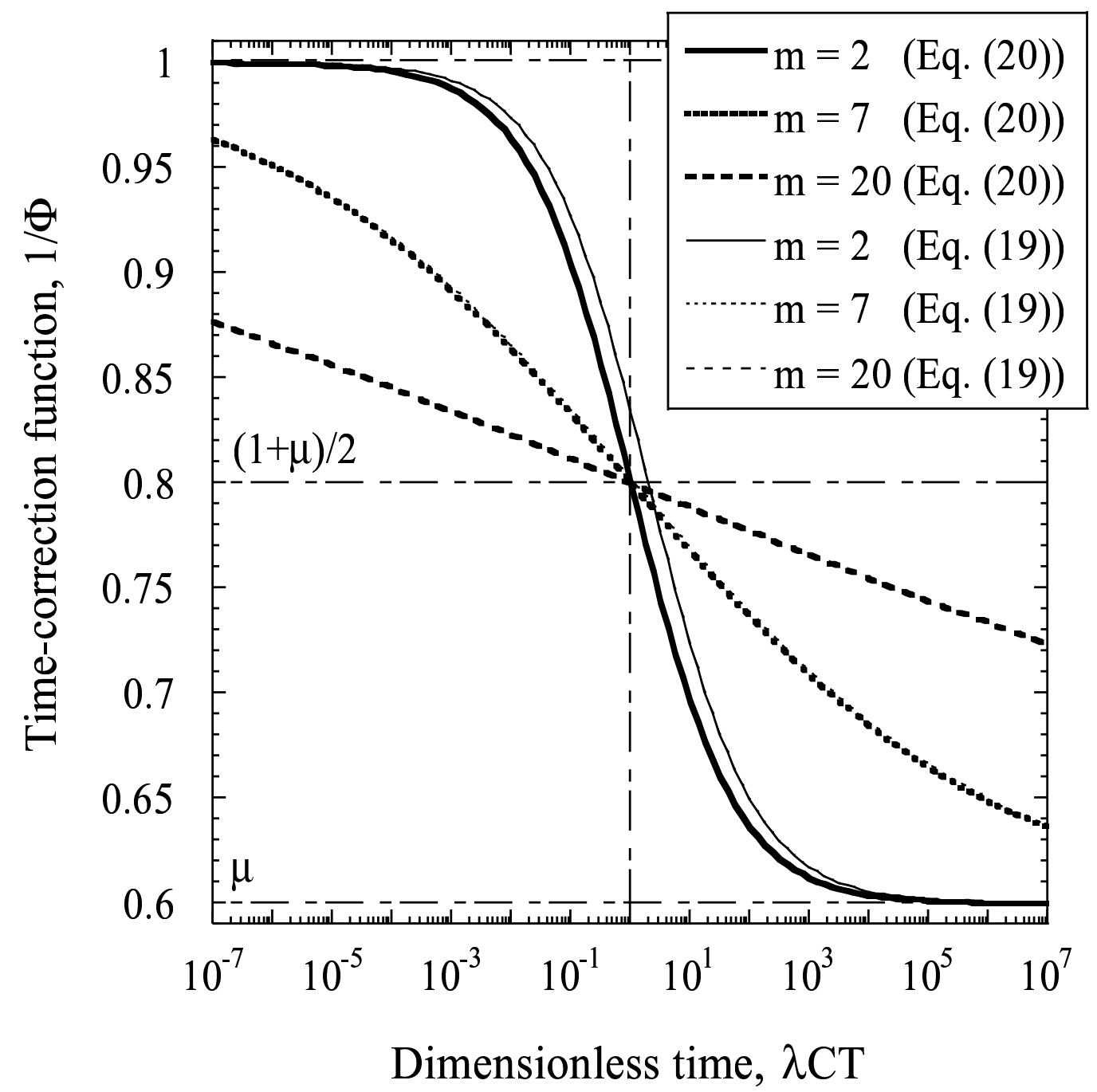

FIG. 2. Time-correction function $\Phi$ vs. dimensionless time $\lambda C T$ for different values of the sensitivity exponent $m$ when $\mu=0.6$.

Charles et al. 




FIG. 3. Identification of the crack propagation parameters $\mu, C, m$ from conventional Evans-Wiederhorn parameters $A, p$ for a $99.6 \%$ alumina ceramic for a conventional value of $V_{0}$.

Charles et al. 


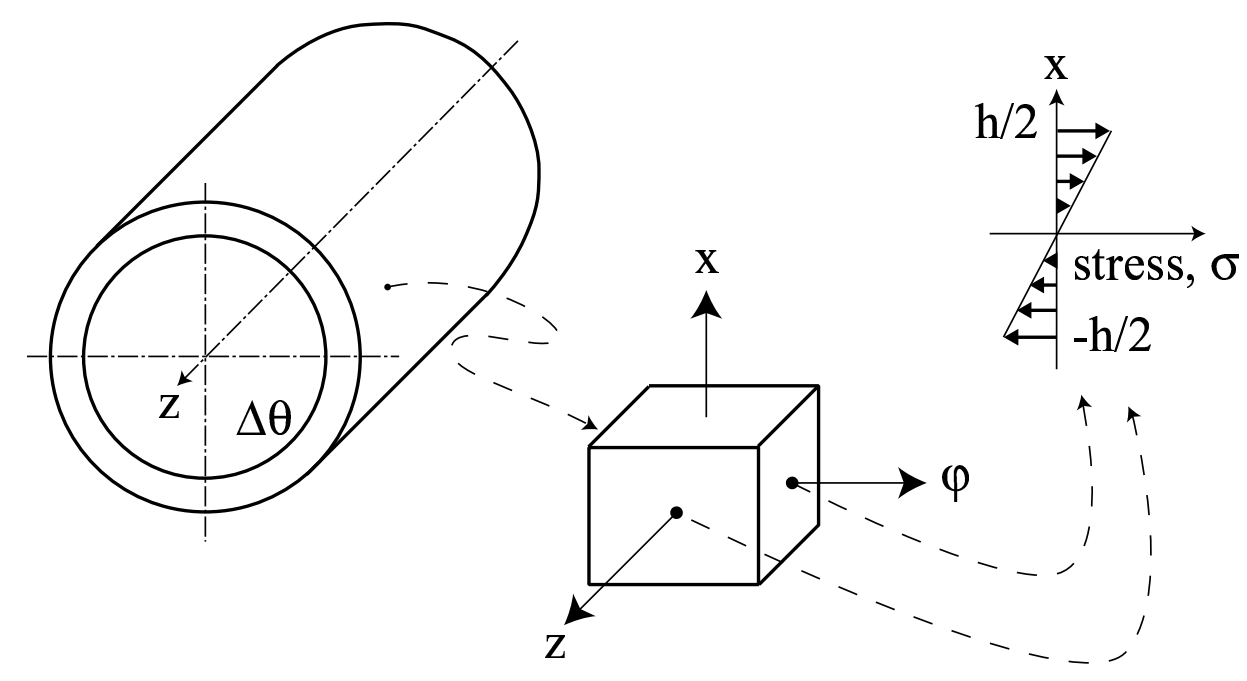

FIG. 4. Thin-walled tube of average radius $R$ and thickness $h$. Frame of a volume element of the tube and distribution of stresses $\sigma_{z z}$ and $\sigma_{\varphi \varphi}$ through the thickness.

Charles et al. 


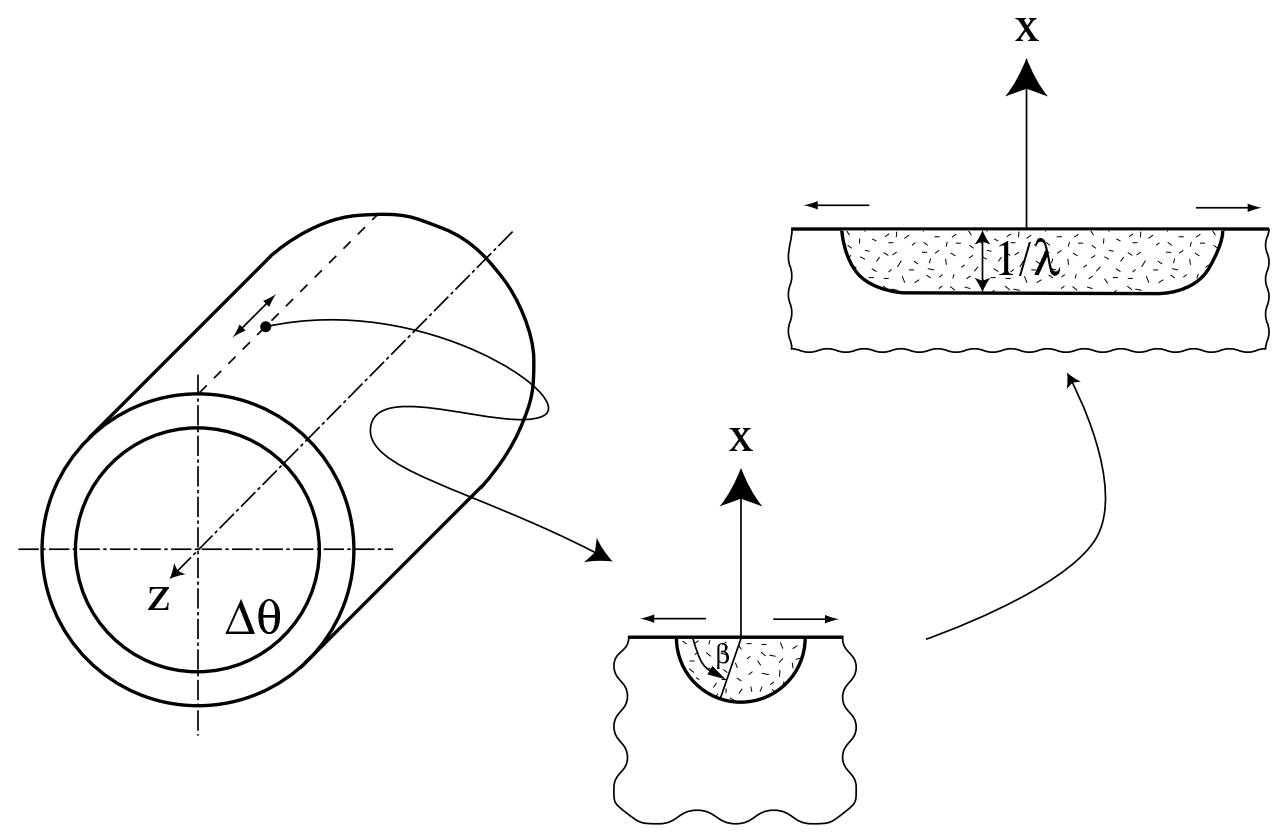

FIG. 5. Longitudinal crack initiated on the outer surface of the tube. Initial semi-circular crack and channelling crack experiencing a steady-state propagation.

Charles et al. 


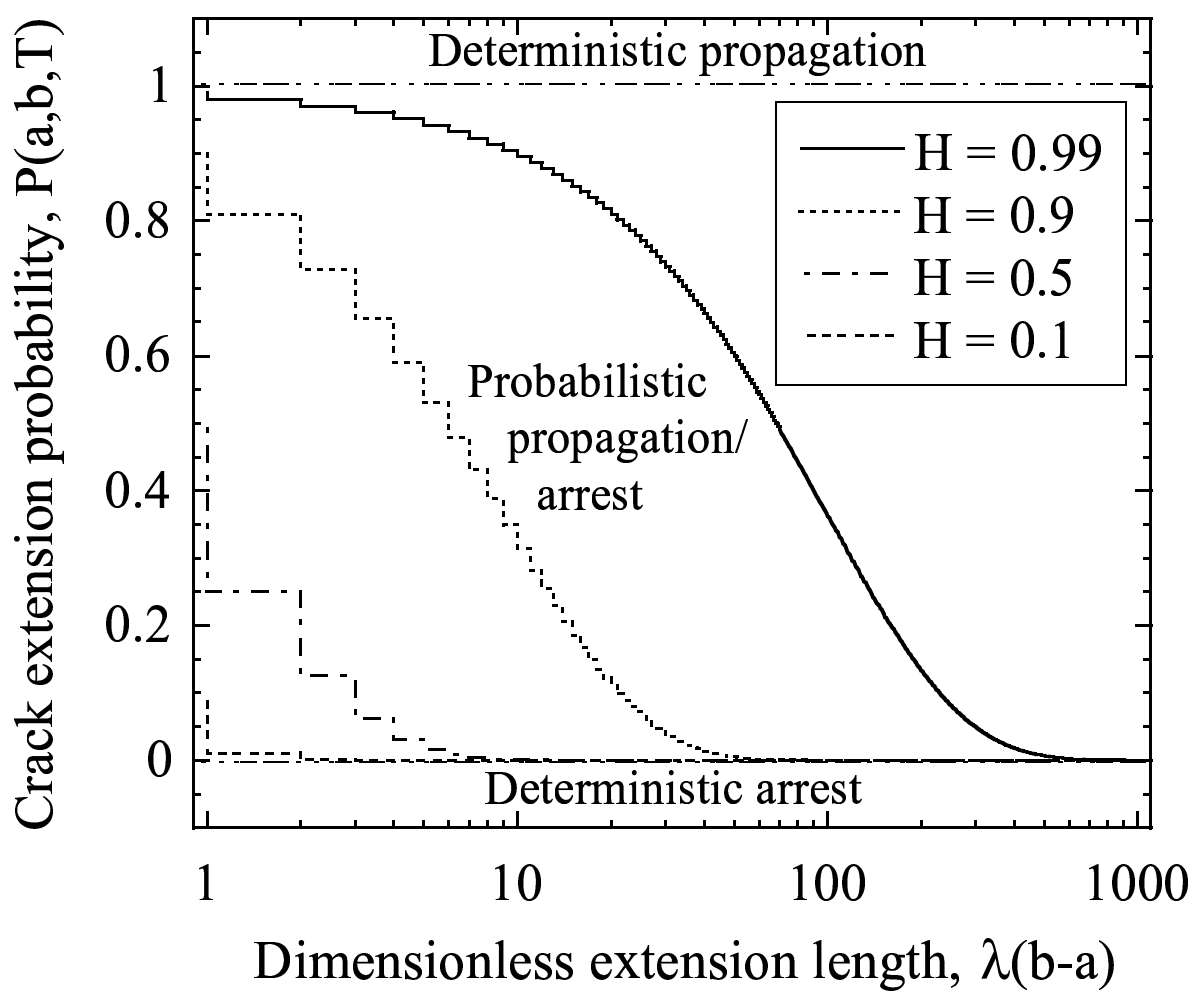

FIG. 6. Crack extension probability vs. dimensionless extension length for different values of the cumulative probability $H\left[K_{s s} \Phi(T)\right]$. Three different regimes can be observed.

Charles et al. 